\title{
Simple targeted assays for metabolic pathways and signaling: a powerful tool for targeted proteomics
}

Dominik Kopczynski ${ }^{1, *}$, Andreas Hentschel ${ }^{1, *}$, Cristina Coman ${ }^{1,2}$, Nils Helge Schebb ${ }^{3,4}$, Thorsten Hornemann ${ }^{5}$, Douglas G. Mashek ${ }^{6,7}$, Nicole M. Hartung ${ }^{4}$, Olga Shevchuk ${ }^{1}$, HansFrieder Schöttt ${ }^{1,8}$, Kristina Lorenz ${ }^{1}$, Federico Torta ${ }^{8}$, Bo Burla ${ }^{9}$, René P. Zahedi ${ }^{10}$, Albert Sickmann $1,11,12$, Michael R. Kreutz ${ }^{13,14,15}$, Christer S. Ejsing ${ }^{16,17}$, Jan Medenbach ${ }^{18}$, Robert Ahrends ${ }^{1,2,+}$

*First author, ${ }^{\dagger}$ Corresponding author (robert.ahrends@univie.ac.at)

1 Leibniz-Institut für Analytische Wissenschaften - ISAS - e.V., Dortmund, Germany

2 University of Vienna, Department of Analytical Chemistry, 1090 Vienna, Austria

3 Institute for Food Toxicology, University of Veterinary Medicine Hannover, Hannover, Germany

4 Chair of Food Chemistry, Faculty of Mathematics and Natural Sciences, University of Wuppertal, Wuppertal, Germany

5 Institute of Clinical Chemistry, University Hospital Zurich, Zurich, Switzerland

6 Division of Diabetes, Endocrinology and Metabolism, Department of Medicine, University of Minnesota, Minneapolis, MN, USA

7 Department of Biochemistry, Molecular Biology and Biophysics, University of Minnesota, Minneapolis, MN, USA

8 Singapore Lipidomics Incubator (SLING), Department of Biochemistry, YLL School of Medicine, National University of Singapore, Singapore

9 Singapore Lipidomics Incubator (SLING), Life Sciences Institute, National University of Singapore, Singapore

10 Segal Cancer Proteomics Centre, Lady Davis Institute, Jewish General Hospital, McGill University, Montréal, Quebec, Canada

11 Medizinische Fakultät, Medizinisches Proteom-Center (MPC), Ruhr-Universität Bochum, Bochum, Germany

12 Department of Chemistry, College of Physical Sciences, University of Aberdeen, Aberdeen, Scotland, United Kingdom

13 Leibniz Group 'Dendritic Organelles and Synaptic Function', University Medical Center Hamburg-Eppendorf, Center for Molecular Neurobiology, ZMNH, Hamburg, Germany

14 RG Neuroplasticity, Leibniz Institute for Neurobiology, Magdeburg, Germany

15 German Center for Neurodegenerative Diseases (DZNE), Magdeburg, Germany

16 Department of Biochemistry and Molecular Biology, University of Southern Denmark, Odense M, Denmark

17 Cell Biology and Biophysics Unit, European Molecular Biology Laboratory, Heidelberg, Germany

18 Institute of Biochemistry I, University of Regensburg, Regensburg, Germany

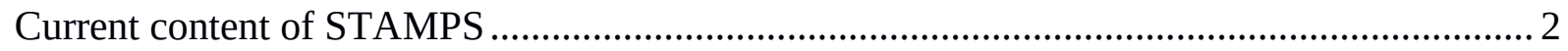

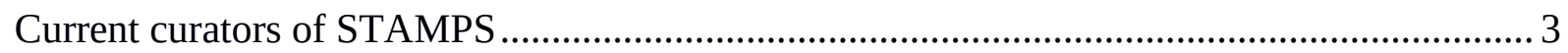

Overview of assay development time for insulin signaling (including 140 proteins) .............. 4

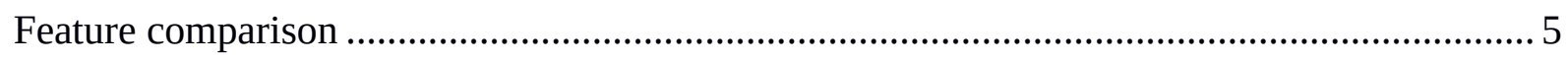

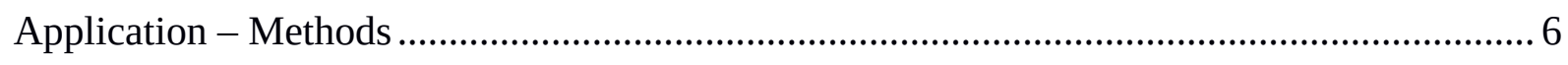



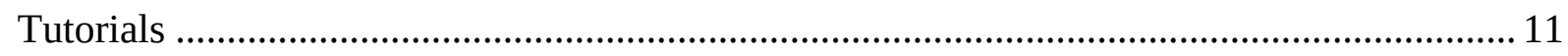

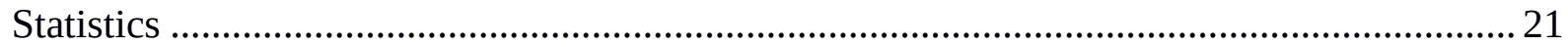




\section{Current content of STAMPS}

Table S1: Current data content of STAMPS

\begin{tabular}{lc}
\hline Registered organisms & $\begin{array}{c}\text { Mouse } \\
\text { Registered tissues }\end{array}$ \\
$\begin{array}{lc}\text { Number of metabolic pathways } \\
\text { Kidney, Liver, Lung, Spleen }\end{array}$ \\
Number of signaling pathways & 56 \\
Number of proteins & 49 \\
Number of unique peptides & 16,810 \\
Number of spectra & 116,873 \\
Number of proteins in pathways & 152,000 \\
Number of PRM / SRM validated & 3,505 \\
Peptides & 670 \\
\hline
\end{tabular}

Information about present pathways and the pathway structure including the protein and metabolite node location and connection were initially extracted from KEGG database ${ }^{1}$, UniProt $^{2}$ and $\mathrm{NCBI}^{3}$.

Table S2: Distribution of identified peptides / spectra among the different tissues. Take into account that there are overlaps of peptides / spectra between the tissues.

\begin{tabular}{|c|c|c|c|c|c|}
\hline tissue & peptides & spectra & tissue & peptides & spectra \\
\hline Blood & 22058 & 25933 & Heart & 13432 & 16557 \\
\hline Brain & 79128 & 98698 & Kidney & 41385 & 52447 \\
\hline Eye & 14145 & 16662 & Liver & 23043 & 28791 \\
\hline Fat & 62454 & 76789 & Lung & 30925 & 38341 \\
\hline Gut & 7476 & 8186 & Spleen & 35144 & 41565 \\
\hline
\end{tabular}




\section{Current curators of STAMPS}

Table S3: current pathway curators of STAMPS

\begin{tabular}{lcc}
\hline $\begin{array}{l}\text { Curated pathway } \\
\text { Adrenergic signaling in } \\
\text { cardiomyocytes }\end{array}$ & Curator & \multicolumn{1}{c}{ Institute } \\
Arachidonic acid metabolism & $\begin{array}{c}\text { Prof. Dristina Lorenz } \\
\text { Nicole M. Hartung, PhD }\end{array}$ & ISAS Dortmund \\
Glycerolipid metabolism & Douglas Mashek, PhD & University of Wuppertal \\
Glycerophospholipid metabolism & Prof. Christer S. Ejsing & University of southern Denmark \\
Glycosphingolipid biosynthesis - & Federico Torta, PhD; Bo & National University of Signapore \\
ganglio series & Burla, PhD & \\
Glycosphingolipid biosynthesis - & Federico Torta, PhD; Bo & National University of Signapore \\
globo and isoglobo series & Burla, PhD & \\
Glycosphingolipid biosynthesis - & Federico Torta, PhD; Bo \\
lacto and neolacto series & Burla, PhD & National University of Signapore \\
Immunological pathways & Dr. Olga Shevchuk & ISAS Dortmund \\
PPAR signaling pathway & Dr. Robert Ahrends & ISAS Dortmund \\
Sphingolipid metabolism & Prof. Thorsten Hornemann \\
Steroid biosynthesis & Dr. Hans-Frieder Schött \\
Steroid hormone synthesis & Dr. Hans-Frieder Schött & University Hospital Zurich \\
UPR signaling & Dr. Jan Medenbach & ISAS Dortmund \\
\hline
\end{tabular}




\section{Overview of assay development time for insulin signaling (including 140 proteins)}

Table S4: time comparison of assay development time between STAMPS and other databases. All time specifications are expressed as hh:mm:ss.

\begin{tabular}{|c|c|c|c|}
\hline $\begin{array}{c}\text { Tested proteomics } \\
\text { pipeline }\end{array}$ & Single steps & $\begin{array}{c}\text { Time for each } \\
\text { step }\end{array}$ & $\begin{array}{c}\text { Complete time } \\
\text { needed }\end{array}$ \\
\hline STAMPS & download assay & $00: 00: 32$ & $00: 00: 32$ \\
\hline PeptideAtlas & $\begin{array}{l}\text { download FASTA file } \\
\text { download of protein library }\end{array}$ & $\begin{array}{l}01: 18: 06 \\
00: 00: 53\end{array}$ & 01:18:59 \\
\hline ProteomicsDB & $\begin{array}{l}\text { download FASTA file } \\
\text { create spectral library }\end{array}$ & $\begin{array}{c}01: 18: 06 \\
00: 40: 30 \cdot n\end{array}$ & $\begin{array}{l}01: 18: 06+ \\
00: 40: 30 \cdot n\end{array}$ \\
\hline Picky & $\begin{array}{l}\text { download FASTA file } \\
\text { download assay }\end{array}$ & $\begin{array}{l}01: 18: 06 \\
00: 01: 23\end{array}$ & 01:19:29 \\
\hline NIST library & $\begin{array}{l}\text { download FASTA file }{ }^{a} \\
\text { download of protein library }\end{array}$ & $\begin{array}{l}01: 18: 06 \\
00: 01: 39\end{array}$ & 01:19:45 \\
\hline
\end{tabular}

a Downloading enzyme list from KEGG (00:03:02), converting KEGG ID to Uniprot accession numbers (01:13:53) and downloading Fasta file from Uniprot (00:01:11)

${ }^{b}$ Including downloading the raw file $(00: 01: 38 \cdot n)$, converting $(00: 20: 57 \cdot n)$, peptide identification with SearchGUI (00:11:05 - n), protein inference with PeptideShaker (00:05:02 - n) and spectral library creation with Skyline (00:01:48 $\cdot n)$, where $n$ is the number of files taken to create the spectral library

The time evaluation is based on a domain expert using all mentioned tools and frameworks to create a fasta file and a spectral library containing all necessary protein information of the insulin signaling pathway. Note, that for PeptideAtlas \& NIST library only complete spectral libraries for mouse could be downloaded as well as a complete library was created when using raw files from ProteomicsDB. In Picky, only $\leq 100$ IDs can be used in one assay. When only human experiments were available in those databases, we took human instead of mouse data. 


\section{Feature comparison}

Table S5: Feature comparison between STAMPS and other databases offering spectral data

\begin{tabular}{|c|c|c|c|c|c|}
\hline & STAMPS & PeptideAtlas $^{1}$ & Proteomics $D B^{2}$ & Picky ${ }^{3}$ & NIST Library ${ }^{4}$ \\
\hline human curation of spectra & $\mathbf{x}$ & $\mathrm{n} / \mathrm{a}$ & $\mathrm{n} / \mathrm{a}$ & $\mathrm{n} / \mathrm{a}$ & $\mathrm{n} / \mathrm{a}$ \\
\hline pathway curation by domain experts & $\mathbf{x}$ & & & & \\
\hline statistical support for method dev. & $\mathbf{x}$ & & & $x$ & \\
\hline web-editor for editing pathways & $\mathbf{x}$ & & & & \\
\hline high-resolution spectra & $\mathbf{x}$ & & $\mathbf{x}$ & & \\
\hline downloadable spectra & $\mathbf{x}$ & $\mathbf{x}$ & & $\mathbf{x}$ & $\mathbf{x}$ \\
\hline protein selection & $\mathbf{x}$ & & & $\mathbf{x}$ & \\
\hline downloadable protein sequence & $\mathbf{x}$ & $\mathbf{x}$ & $\mathbf{x}$ & $\mathbf{x}$ & \\
\hline peptide selection & $\mathbf{x}$ & & & & \\
\hline peptides from real data & $\mathbf{x}$ & & $\mathbf{x}$ & & $\mathbf{x}$ \\
\hline spectra selection & $\mathbf{x}$ & & & & \\
\hline spectrum view & $\mathbf{x}$ & & $\mathbf{x}$ & $\mathbf{x}$ & \\
\hline mouse data & $\mathbf{x}$ & $\mathbf{x}$ & & $\mathbf{x}$ & $\mathbf{x}$ \\
\hline human data & $(\mathbf{X})$ & $\mathbf{x}$ & $x$ & $\mathbf{x}$ & $x$ \\
\hline proteins tissue resolved & $x$ & & $x$ & & \\
\hline proteins chromosome resolved & $\mathbf{x}$ & $\mathbf{x}$ & $\mathbf{x}$ & & \\
\hline proteins pathway resolved & $\mathbf{x}$ & & & & \\
\hline visual protein selection & $\mathbf{x}$ & & & & \\
\hline protein batch selection & $\mathbf{x}$ & & & $\mathbf{x}$ & \\
\hline proteins enzymatically resolved & $\mathbf{x}$ & & $\mathbf{x}$ & & \\
\hline proteins subcellular resolved & $\mathbf{x}$ & & $\mathbf{x}$ & & \\
\hline assay development & $\mathbf{x}$ & & & $\mathbf{x}$ & \\
\hline integration with cons. analysis tool ${ }^{*}$ & $\mathbf{x}$ & & & $\mathbf{x}$ & \\
\hline
\end{tabular}

\footnotetext{
${ }^{1}$ http://www.peptideatlas.org/

2 https://www.proteomicsdb.org/

${ }^{3}$ https://picky.mdc-berlin.de/

${ }^{4}$ https://peptide.nist.gov/
} 


\section{Application - Methods}

\section{Cell culture}

Preadipocytes were cultivated in minimum essential medium (Mem- $\alpha$ ) supplemented with $20 \%$ fetal bovine serum (FBS) and $1 \%$ penicillin/streptomycin (PSG) at $37^{\circ} \mathrm{C}$ and $5 \% \mathrm{CO}_{2}$. Medium change was done every two days followed by propagation with trypsin if cells were at $80 \%$ confluence. The experiment consisted out of 4 different conditions: control, induced insulin sensitivity, induced insulin resistance and both together. For each of the 4 conditions, cells were grown until $80 \%$ of confluence to start the treatment. For inducing insulin sensitivity, cells were treated with $1 \mu \mathrm{M}$ of rosiglitazone for $48 \mathrm{~h}$. To induce insulin resistance, the treatment was done with $10 \mathrm{ng} / \mathrm{ml} \mathrm{TNF} \alpha$ for $24 \mathrm{~h}$. The last condition was prepared by adding first $1 \mu \mathrm{M}$ rosiglitazone. After the incubation time of $48 \mathrm{~h}$, cells were washed and $10 \mathrm{ng} / \mathrm{ml}$ TNF $\alpha$ was added for additional $24 \mathrm{~h}$. The control condition comprised preadipocytes grown to $80 \%$ confluence and treated with just $0.01 \%$ Dimethylsulfoxid (DMSO). Harvested cells were washed with PBS, centrifuged and snap frozen. See Figure S1 for a summarizing flow chart.

\section{Sample preparation}

Cell pellets were lysed with a 1\% SDS lysis buffer. Frozen mouse organs were homogenized by grinding in liquid nitrogen, one at a time. To achieve this, each organ were placed in a liquid nitrogen-cooled ceramic mortar separately. The tissues were manually grinded with a pestle to obtain a fine powder (sterile conditions). The powder was collected in Eppendorf reaction tubes and $50 \mu \mathrm{g}$ of each prepared organ were lysed using a 1\% SDS lysis buffer.

To remove interfering DNA, the samples were treated with Benzonase ${ }^{\circledR}$ for $30 \mathrm{~min}$ at $37^{\circ} \mathrm{C}$, followed by protein precipitation using 3 volumes of ice cold acetone overnight at $-20^{\circ} \mathrm{C}$. Precipitated proteins were harvested by centrifugation at $20.000 \mathrm{~g}$ for $20 \mathrm{~min}$ at $4{ }^{\circ} \mathrm{C}$ and dissolved in $100 \mu \mathrm{l} 8 \mathrm{M}$ Urea for $3 \mathrm{~h}$ at room temperature (RT). Protein amount was checked by conducting a BCA according to the manufacturer's instructions. Samples were reduced by adding $10 \mathrm{mM}$ tris(2-carboxyethyl)phosphine (TCEP) for $30 \mathrm{~min}$ at $37^{\circ} \mathrm{C}$ and alkylated by adding $15 \mathrm{mM}$ of iodoacetamide (IAA) for $30 \mathrm{~min}$ at $21^{\circ} \mathrm{C}$ (dark). Hydrolysis of proteins was done overnight at $37^{\circ} \mathrm{C}$ using a trypsin to protein ratio of $1: 100$. The reaction was stopped by adding $2 \mu \mathrm{l}$ of formic acid (FA, 99\%). The samples were desalted using solid phase extraction with C18 filter cartridges, washed with $0.1 \%$ trifluoroacetic acid (TFA) and eluted with $80 \%$ 
acetonitrile (ACN). Cleaned samples were dried by using a vacuum concentrator. Concentration was adjusted to $1 \mu \mathrm{g} / \mu \mathrm{l}$ with $0.1 \%$ TFA. Proteolytic digests were checked for complete digestion after desalting by using monolithic column separation (PepSwift monolithic PS-DVB PL-CAP200-PM, Dionex) on an inert Ultimate 3000 HPLC (Dionex, Germering, Germany) by direct injection of $1 \mu \mathrm{g}$ sample. A binary gradient (solvent A: $0.1 \%$ TFA, solvent $\mathrm{B}: 0.08 \%$ TFA, $84 \% \mathrm{ACN}$ ) ranging from $5-12 \% \mathrm{~B}$ in $5 \mathrm{~min}$ and then from $12-50 \% \mathrm{~B}$ in $15 \mathrm{~min}$ at a flow rate of $2.2 \mathrm{\mu L} / \mathrm{min}$ and at $60^{\circ} \mathrm{C}$, was applied. UV traces were acquired at $214 \mathrm{~nm}$.

For $\mathrm{pH} 8$ fractionation each of the digested and desalted samples was first dried using a vacuum concentrator. Subsequently, the peptides were brought back into solution in a buffer consisting of $10 \mathrm{mM}$ ammonium acetate and $0.4 \mathrm{mM}$ formiate $(\mathrm{pH} 8.0)$ and separated on a C18 RP column $(50 \mu \mathrm{g})$. The separation and fractionation was performed with the following gradient by solvent $\mathrm{B}$ ( $84 \%$ acetonitrile in $10 \mathrm{mM}$ ammonium acetate, $0.4 \mathrm{mM}$ formiate, $\mathrm{pH}$ 8.0): 3-10\% B for $10 \mathrm{~min}, 10-25 \%$ B for $35 \mathrm{~min}, 25-40 \%$ for $20 \mathrm{~min}$, $40-95 \%$ for $10 \mathrm{~min}$., $95 \%$ for $5 \mathrm{~min}$. and $20 \mathrm{~min}$. equilibration at $3 \% \mathrm{~B}$. The individual fractions were collected in a time interval of $60 \mathrm{~s}$, each sample being divided into 16 fractions. The fractions were collected in concatenated manner. After fractionation, the individual samples were dried in a vacuum concentrator and recorded in $0.1 \%$ TFA $(1 \mu \mathrm{g} / \mu \mathrm{l})$ for the subsequent nano LC-MS/MS analysis.

\section{Method development and targeted LC-MS analysis}

To develop the method for the targeted LC-MS/MS analysis, we used STAMPS to select proteins and peptides for the tricarboxylic acid (TCA) cycle and insulin signaling pathway according to the pathway browser and the built spectral library. After importing the method into Skyline, three transitions were chosen for each peptide that showed the best signal to noise ratio. In addition, synthetic peptides were synthesized for each chosen proteotypic peptide to avoid false positive peak annotation and to confirm the correct retention time. Synthetic peptides were labeled with $\left[{ }^{13} \mathrm{C}_{6}\right] \mathrm{R}$ and $\left[{ }^{13} \mathrm{C}_{6}\right] \mathrm{K}$. After creating a scheduled SRM method with 5 minute windows, we were able to scan for the proteins of one whole pathway per measurement by keeping the maximum concurrent occurring transitions always below 100. 
Chromatographic separation of peptides was achieved on an UltiMate 3000 RSLC nano System

(Dionex) using a $75 \mu \mathrm{m} \times 2 \mathrm{~cm} \mathrm{C18} \mathrm{pre} \mathrm{column} \mathrm{coupled} \mathrm{to} \mathrm{a} 75 \mu \mathrm{m} \times 50 \mathrm{~cm}$ C18 reversed phase main column (Acclaim Pepmap, Thermo Scientific). A 30 min gradient (0.1\% FA, $2-35 \%$ ACN) was applied with a flow speed of $250 \mathrm{nl} / \mathrm{min}$. Peptides were ionized by using a nano spray ESIsource and analyzed on a TSQ Vantage mass spectrometer.
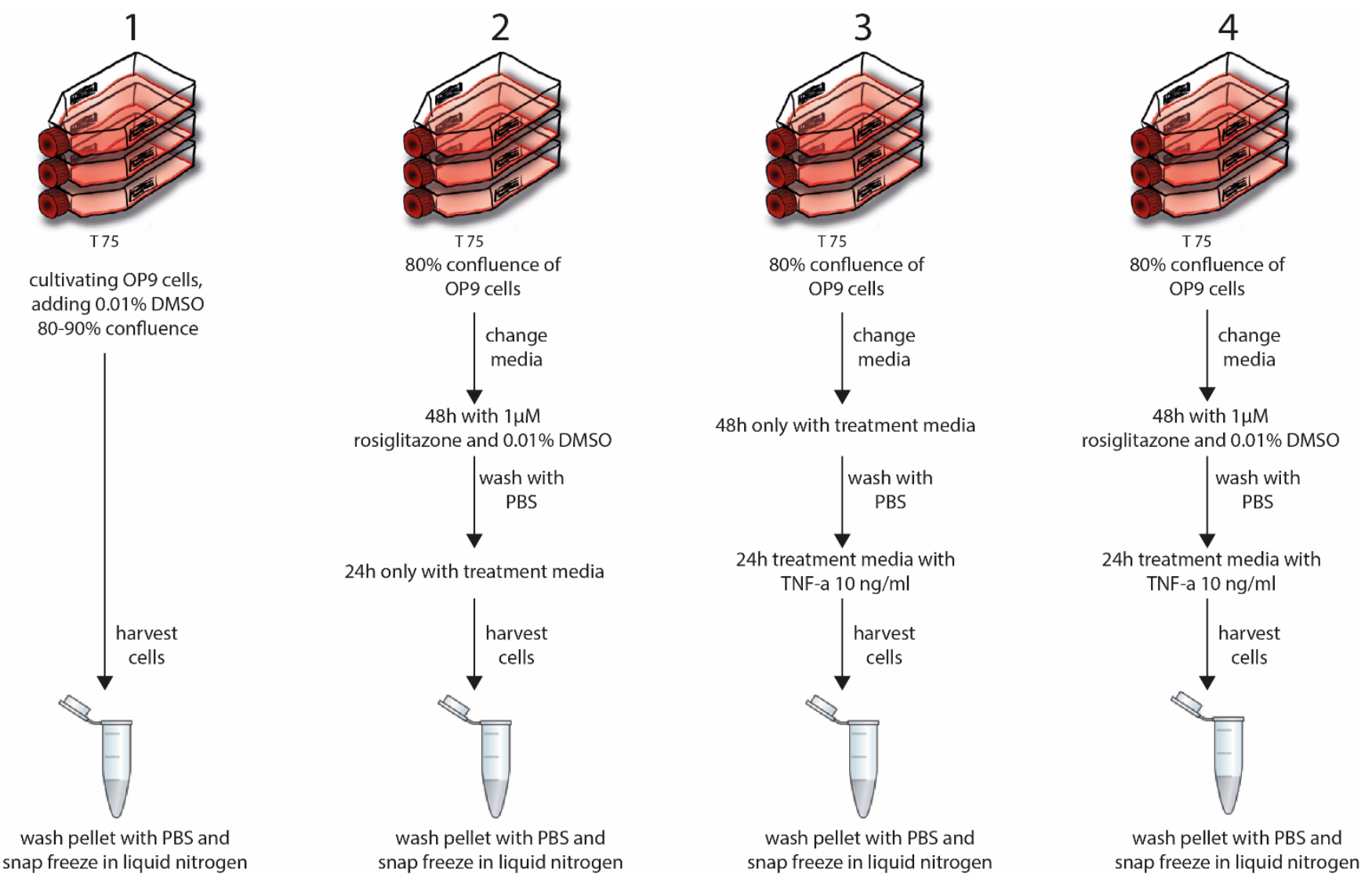

Figure S1 I Summarizing flow chart of the different steps for sample preparation. 1: Control condition comprising only normal grown preadipocytes for a period of $72 \mathrm{~h}$ containing $0.01 \%$ of DMSO. 2: Steps to prepare insulin-resistant cells by treating them with $10 \mathrm{ng} / \mathrm{ml} \mathrm{TNF \alpha}$. 3: Steps to prepare insulin sensitive cells by treating them with $1 \mu \mathrm{M}$ of rosiglitazone for $48 \mathrm{~h}$. 4: Combination of treatments to check if TNF $\alpha$ is able to revert the effect of rosiglitazone.

\section{Application - Results}

To determine the practicability of the workflow, we compared STAMPS with state-of-the-art targeted proteomics tools (Table S4). We measured the time to create a targeted assay for insulin-signaling. Here, a time reduction of $\gtrsim 150$-fold was achieved compared to the second fastest tool. A list of all features in comparison to other state-of-the-art tools is shown in Table S5.

Next, to confirm the applicability of STAMPS, we selected insulin-signaling and the tricarboxylic acid cycle (TCA), two pathways important for the overall metabolism of higher 
eukaryotes. Key proteins of these pathways were analyzed after inducing insulin resistance and sensitivity (Figure 2, S1 - S3).
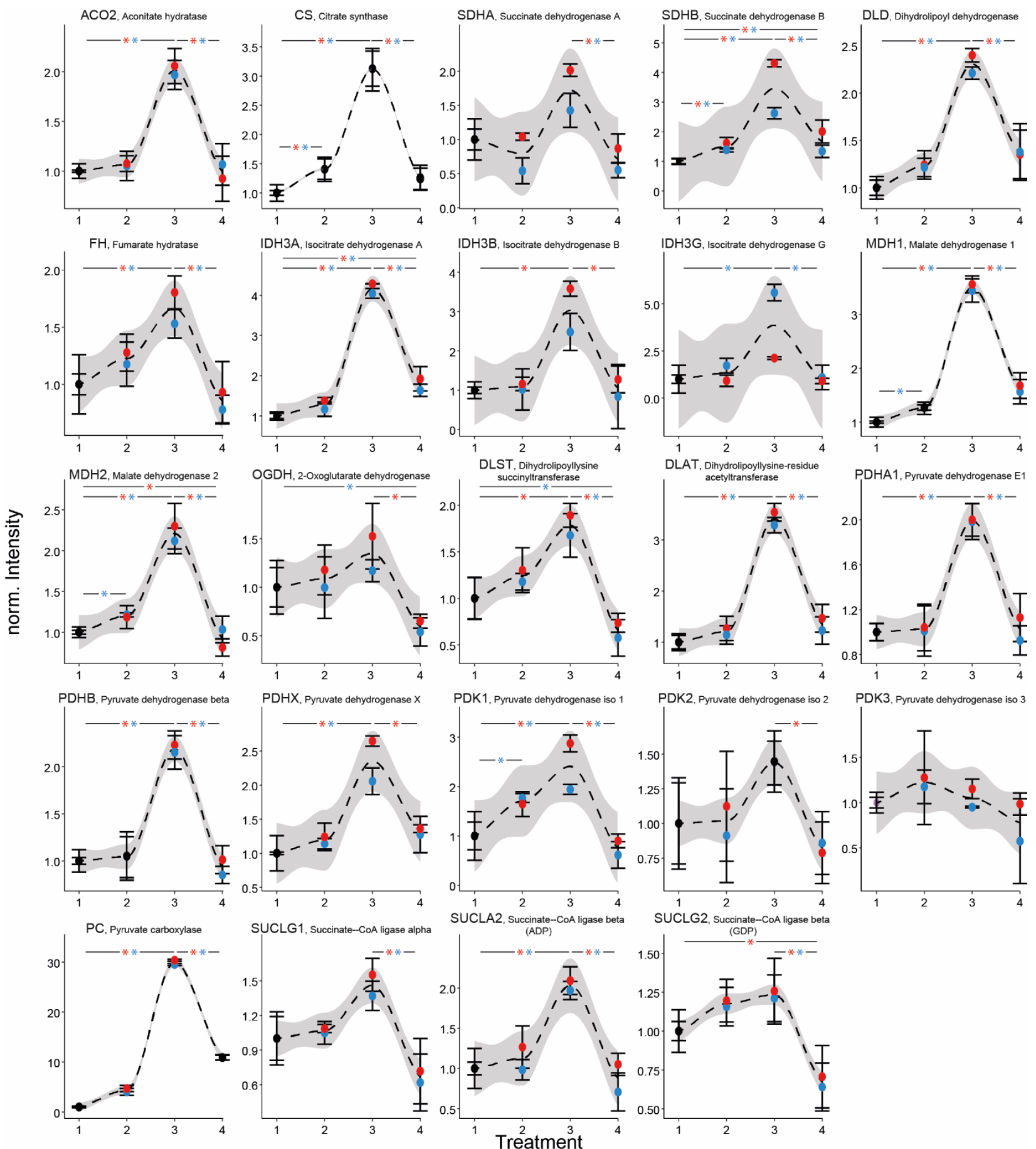

Figure S2 / Overview of all measured proteins part of the TCA. In total 24 proteins of the TCA were measured. Colors represent the two unique peptides used per protein for the targeted analysis. Standard deviation was calculated with 3 biological replicates of each condition $(n=3)$. Numbers represent the different treatments: (1) DMSO control, (2) $10 \mathrm{ng} / \mathrm{ml} \mathrm{TNF \alpha ,} \mathrm{(3)} 1 \mu \mathrm{M}$ rosiglitazone, (4) initial $48 \mathrm{~h}$ rosiglitazone plus $24 \mathrm{~h}$ TNF $\alpha$ afterwards. Asterisks were used to show significant changes in the peptide abundance compared to the control or by comparing the last 2 conditions. For better visualization, a dashed black line was added to show the trend of the respective treatment. 


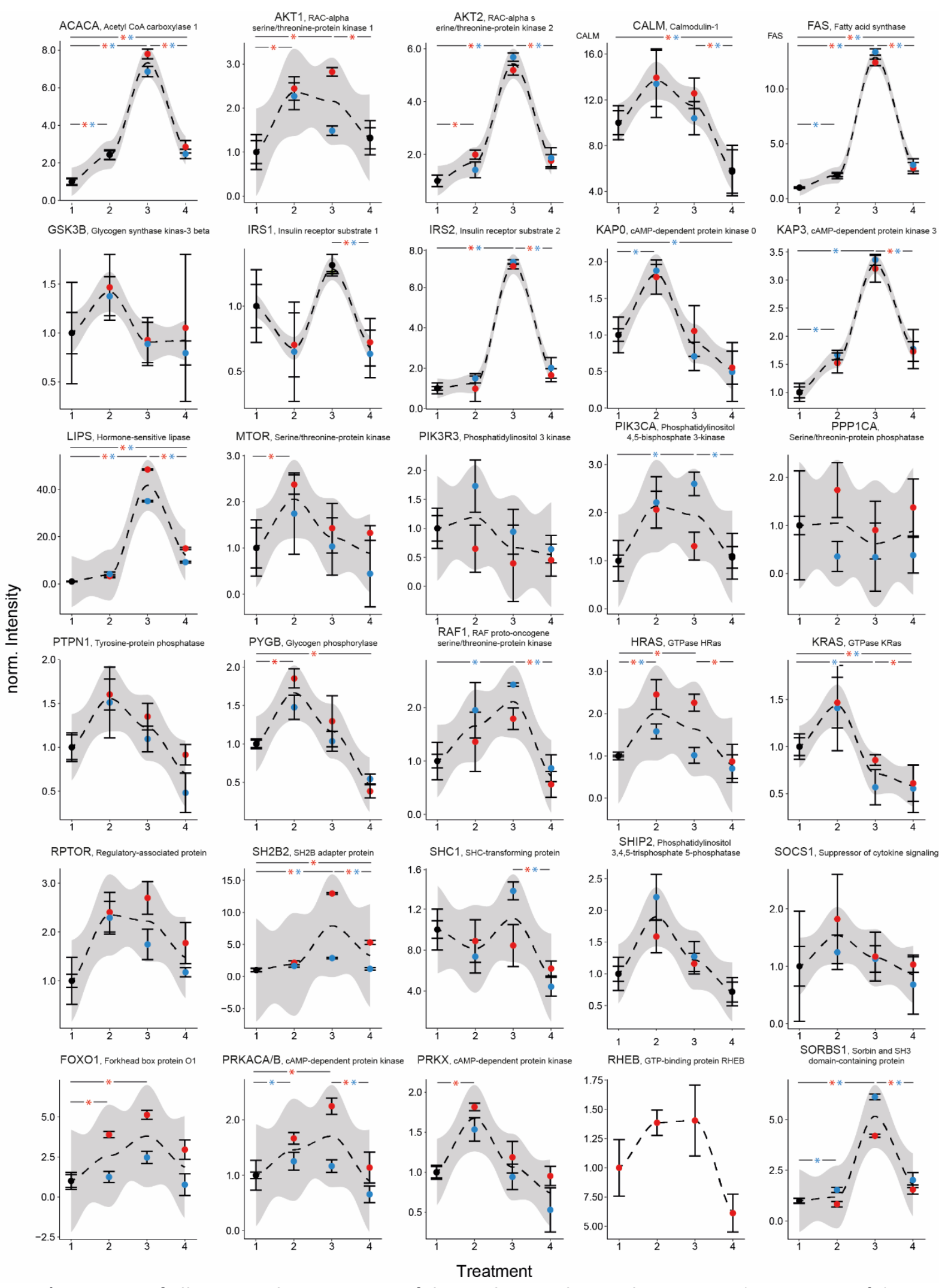

Figure S3 / Overview of all measured proteins part of the insulin signaling pathway. In total 30 proteins of the insulin signaling pathway were measured, representing the key players. Colors represent the two unique peptides used per protein for the targeted analysis. Standard deviation was calculated with 3 biological replicates of each condition $(n=3)$. Numbers represent the different treatments: (1) DMSO control, (2) $10 \mathrm{ng} / \mathrm{ml}$ TNF $\alpha,(3)$ $1 \mu \mathrm{M}$ rosiglitazone, (4) initial $48 \mathrm{~h}$ rosiglitazone plus $24 \mathrm{~h}$ TNF $\alpha$ afterwards. Asterisks where used to show significant changes in the peptide abundance compared to the control or by comparing the last 2 conditions. For better visualization, a dashed black line was added to show the trend of the respective treatment. 


\section{Tutorials}

\section{Creating an assay using the pathway browser}

One of the major tasks in targeted proteomics is building up methods to analyze proteins of one or multiple pathways to uncover their behavior upon different treatments revealing coherencies and patterns, for instance in the metabolism. To facilitate those studies and gain results as shown in Figure 2, STAMPS provides a multitude of intuitive interfaces for fast protein selection, spectra reviewing, assay building and is interlinked with Skyline.

\section{1) Select pathway browser}

The first page of the STAMPS ${ }^{5}$ website is leading to all main protein selection options as visible in Figure S4. Clicking on "Pathway Browser" in the lower panel will lead to the interactive main pathway browser window (Figure S5). The view can be shifted (right click) and the zoom can be changed (mouse wheel or magnifier icons on bottom right position). The metabolic pathways are plotted in a graph-based fashion. Metabolites are presented as circles; proteins which act in the same metabolic reaction are grouped in protein boxes.

By directly clicking on metabolite or protein labels, additional information is displayed.

Links to other Pathways are indicated in round-cornered boxes. By clicking on them, the

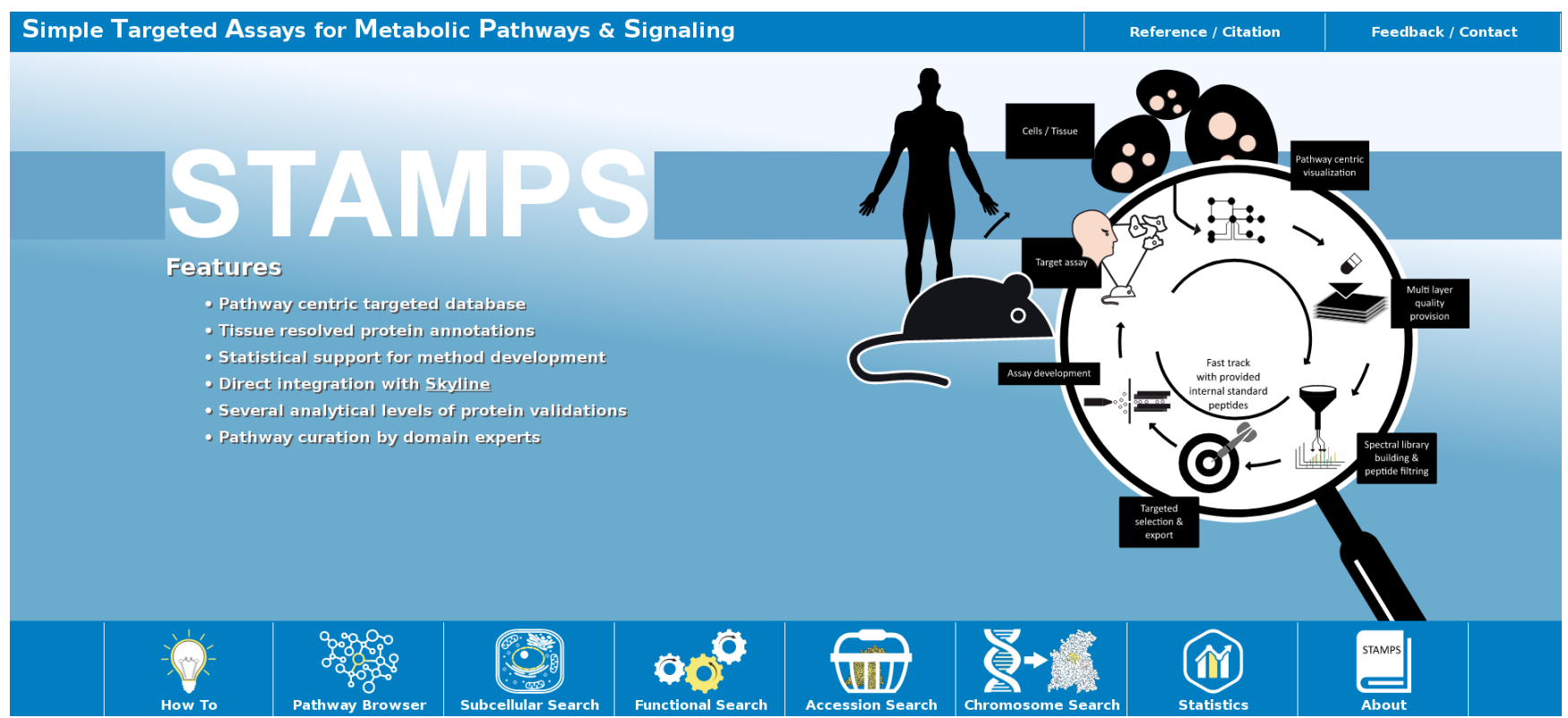

according pathway will be opened.

Figure S4 I Main page of STAMPS providing easy accession to all functions and statistical summaries.

\footnotetext{
${ }^{5}$ https://stamps.isas.de
} 


\section{2) Select pathway(s)}

In the upper panel, the pathway of interest can be chosen. For a better overview, pathways are grouped into metabolic and signaling pathways. Clicking on one of those options will open a drop down menu. Former protein selections are preserved when switching back to a pathway. Additional functions are (i) changing the organism (in the current version, only mouse data is available), (ii) various filters for viewing proteins and (iii) a case insensitive search function for key words like a protein/metabolite name, description, accession number or even peptide sequence. When selecting a protein searched this way, the view is automatically centered and zoomed on the hit in the pathway map.

\section{3) Select proteins}

Proteins of choice can be (de)selected by clicking on their checkboxes. Double-clicking on a protein group box will (de)select all proteins of the according box. By clicking and holding down the left mouse button on a free space and moving the mouse, a protein selection area will be spanned. When releasing the button, all proteins within this selection window will be (de)selected.

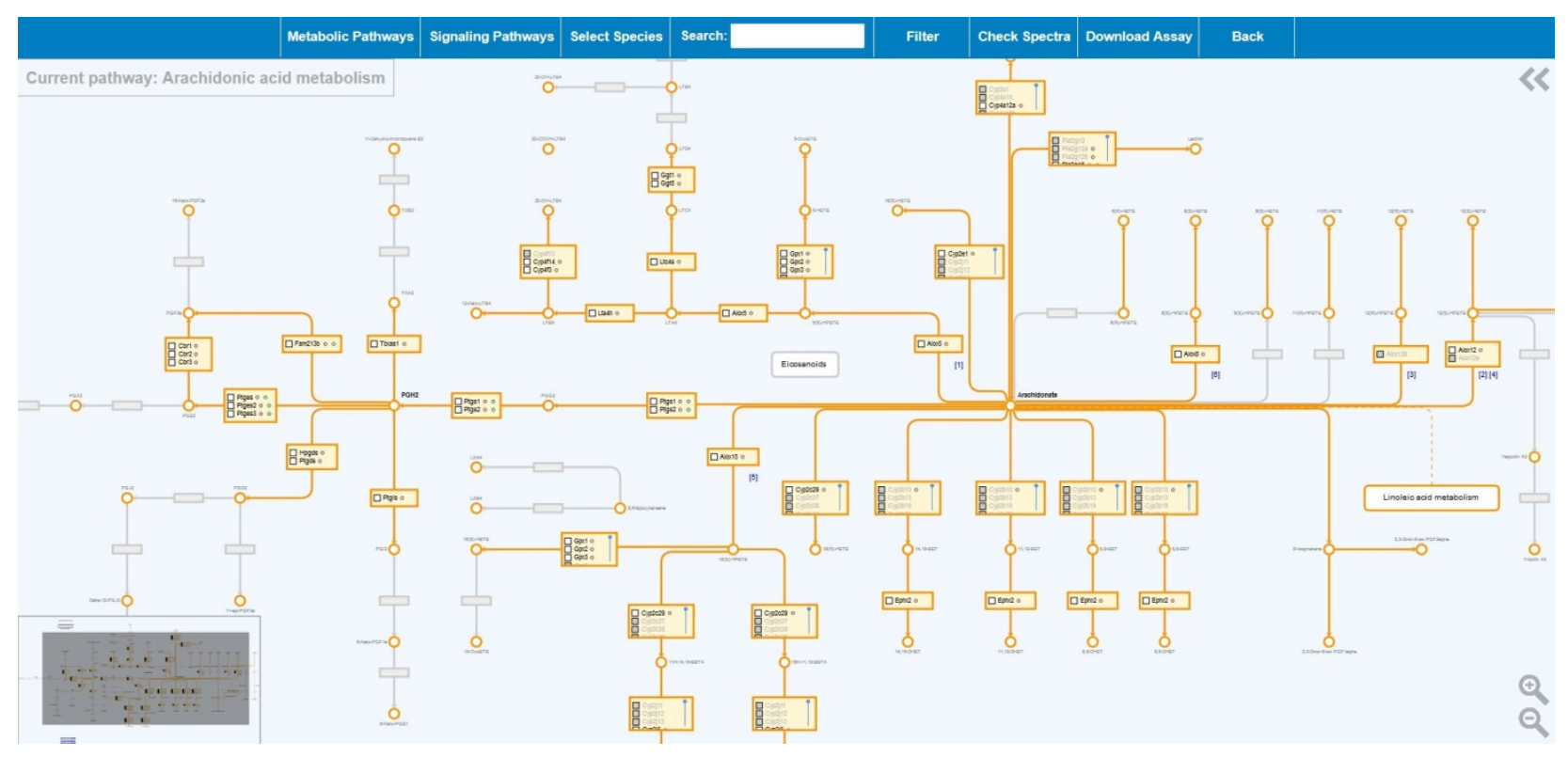

Figure S5 / The interactive pathway browser is the main protein selection window. 
The user has the possibility to get a statistical overview for each pathway by clicking on the arrows in the upper right corner. A window will appear giving information of the total protein number of the selected pathway, protein coverage, number of selected proteins and additional information regarding the number of peptides, spectra and validation (see Figure S6).

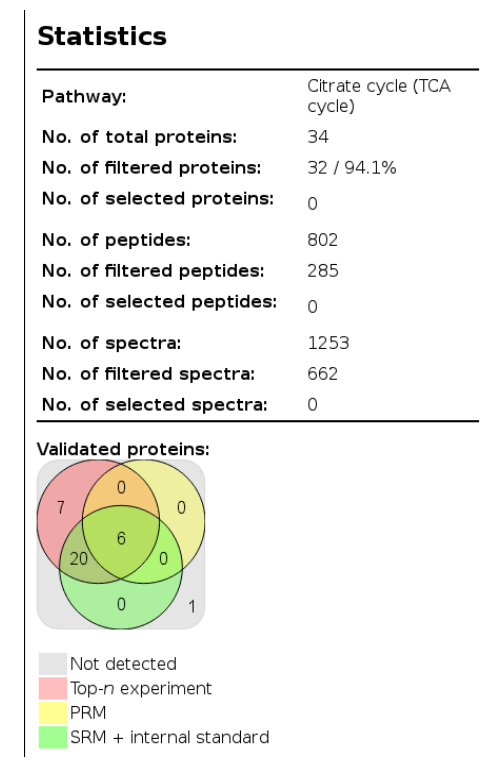

Figure S6 / Statistics box displaying all relevant information for a certain pathway.

\section{5) Check the protein selection and review spectra}

Having selected all proteins for an assay, the user can review the selection by clicking on "Check spectra" in the upper panel. A new window opens as shown in Figure S7. On the left-hand side, a table is showing all selected proteins. The user has the possibility to expand each protein to show its corresponding peptides that are stored with an according spectrum in the database. By clicking on the precursor mass, the corresponding spectrum pops up showing $\mathrm{y}$ and $\mathrm{b}$ ions. All proteins, peptides or spectra can be (de)selected on this screen for the final assay by checking or unchecking the blue boxes. To refine the assay even more, specific filter criteria can be applied. For instance, the user can filter for peptide length and precursor charge as well as choose between variable and fixed modifications of the oxidation of methionine and/or the carbamidomethylation of cysteine. In Addition, there is the possibility to filter for specific tissues. The user has to take into consideration that only proteins, peptides and precursors will be displayed, 
which were identified and stored in our database and in addition satisfy the adjusted filter criteria.

\section{6) Download the assay}

Once the selection is done, the user can proceed to download the assay by clicking on "Go to download" on the bottom right side of the dialog. A spectral library in .blib format, a fasta file containing all selected protein sequences and a Skyline project file are being created on the fly and packed as a .zip file offered for download. After downloading and unzipping, the Skyline project file can easily be opened in Skyline ${ }^{6}$ with the complete assay ready to start the targeted analyses.

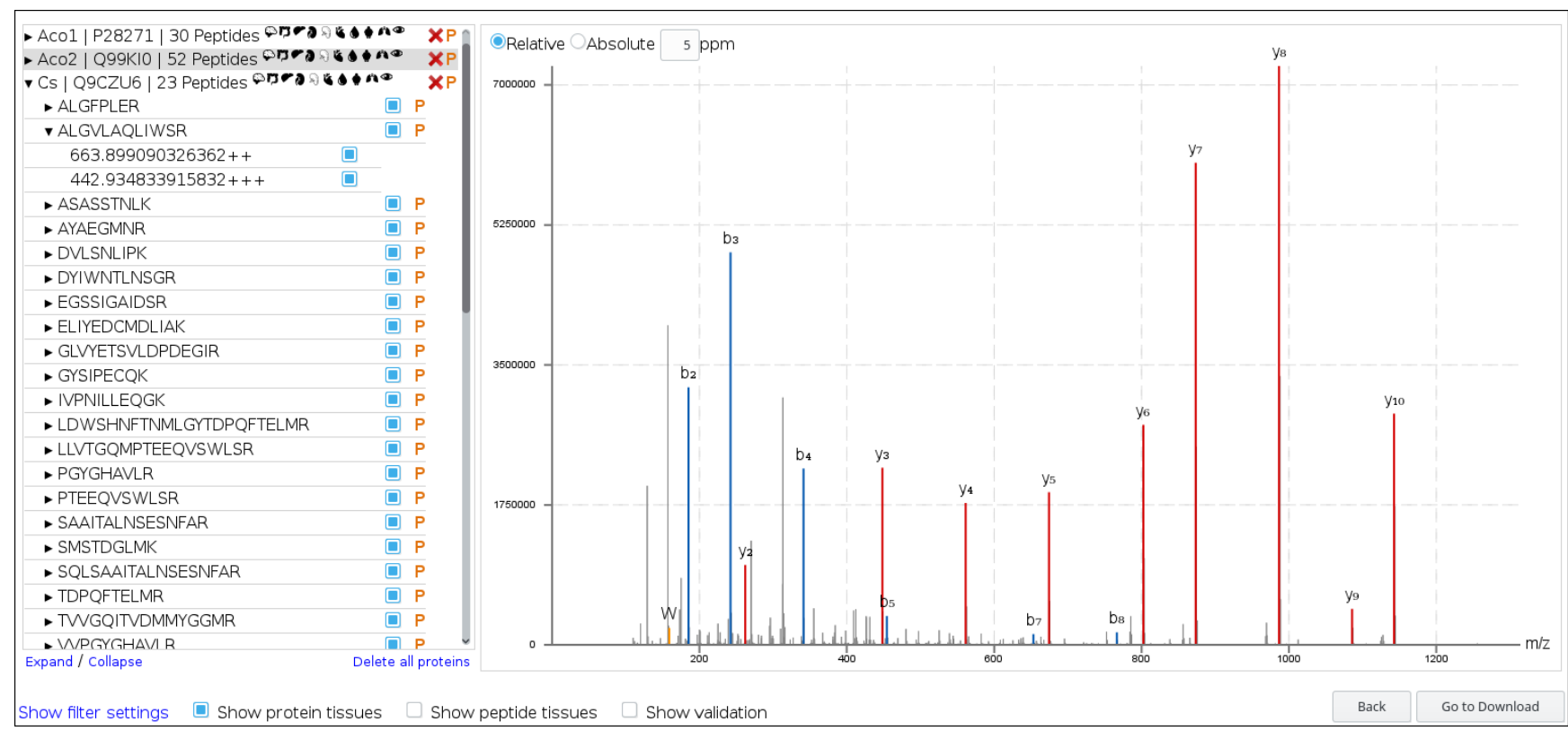

Figure S7 / Review dialog for (de)selecting proteins, peptides or single spectra in the assay.

\section{Creating an assay using the accession list}

\section{1) Open the protein selection window via accession IDs}

For this step, a list of UniProt ${ }^{7}$ accession IDs is required. On the main page (consider Figure S4) one has to click on "Accession Search" in the lower panel.

\footnotetext{
${ }^{6}$ https://skyline.ms

${ }^{7}$ https://www.uniprot.org
} 


\section{2) Paste your protein accession IDs}

A window for inserting the accession IDs is being opened (as seen in Figure S8). The drop down menu is used to select the desired species. The user can type in the accession IDs into the text field. If there is a longer list, it is possible to copy and paste it into the text field. Click on "Next" to proceed.

\section{3) Check the protein selection and review the spectra}

The review dialog appears as known from the previous instructions (Figure S7), proceeding with step 5 of the pathway browser tutorial.

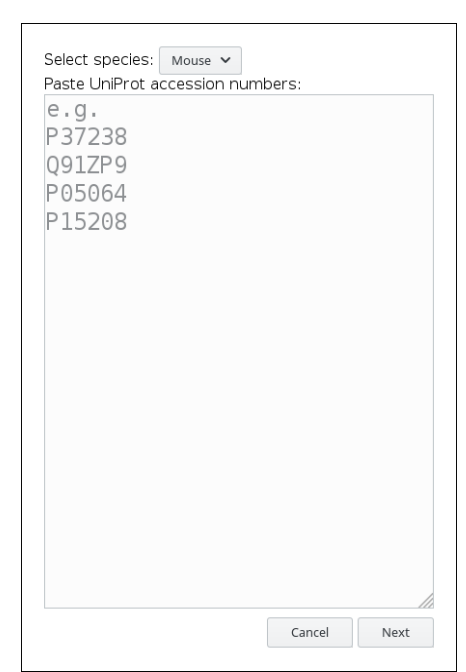

Figure S8 | Dialog for selecting proteins via a list of UniProt accession IDs.

\section{Creating an assay using the chromosome browser}

\section{1) Open protein selection window via chromosome browser}

Another way of building up assays for targeted LC-MS/MS analysis is by selecting proteins of interest according to their gene location on the chromosomes. On the main page (consider Figure S4) click on "Chromosome Search" in the lower panel.

\section{2) Paste your protein accession IDs}

A window for selecting genes within the chromosomes appears (as illustrated in Figure S9). Here, the desired species can be chosen in the upper panel. The user can select different regions on each chromosome by clicking on a specific spot and adjusting the size of the selection box by using the mouse wheel. Alternatively it is possible to drag and drop 
the box along the chromosome or by entering a specific chromosome location in the upper panel.

On the right-hand side, a gene table lists all registered genes available in the particular chromosome region including gene name and accession number. Genes can be (de)selected with the checkboxes to the right. When the selection is complete, one can proceed by clicking on "Next". Only proteins and peptides according to the selected genes that were identified and stored in the background library will be displayed.

\section{3) Check your protein selection and review spectra}

The review dialog appears as known from the previous instructions (Figure S7), proceeding with step 5 of the pathway browser tutorial.

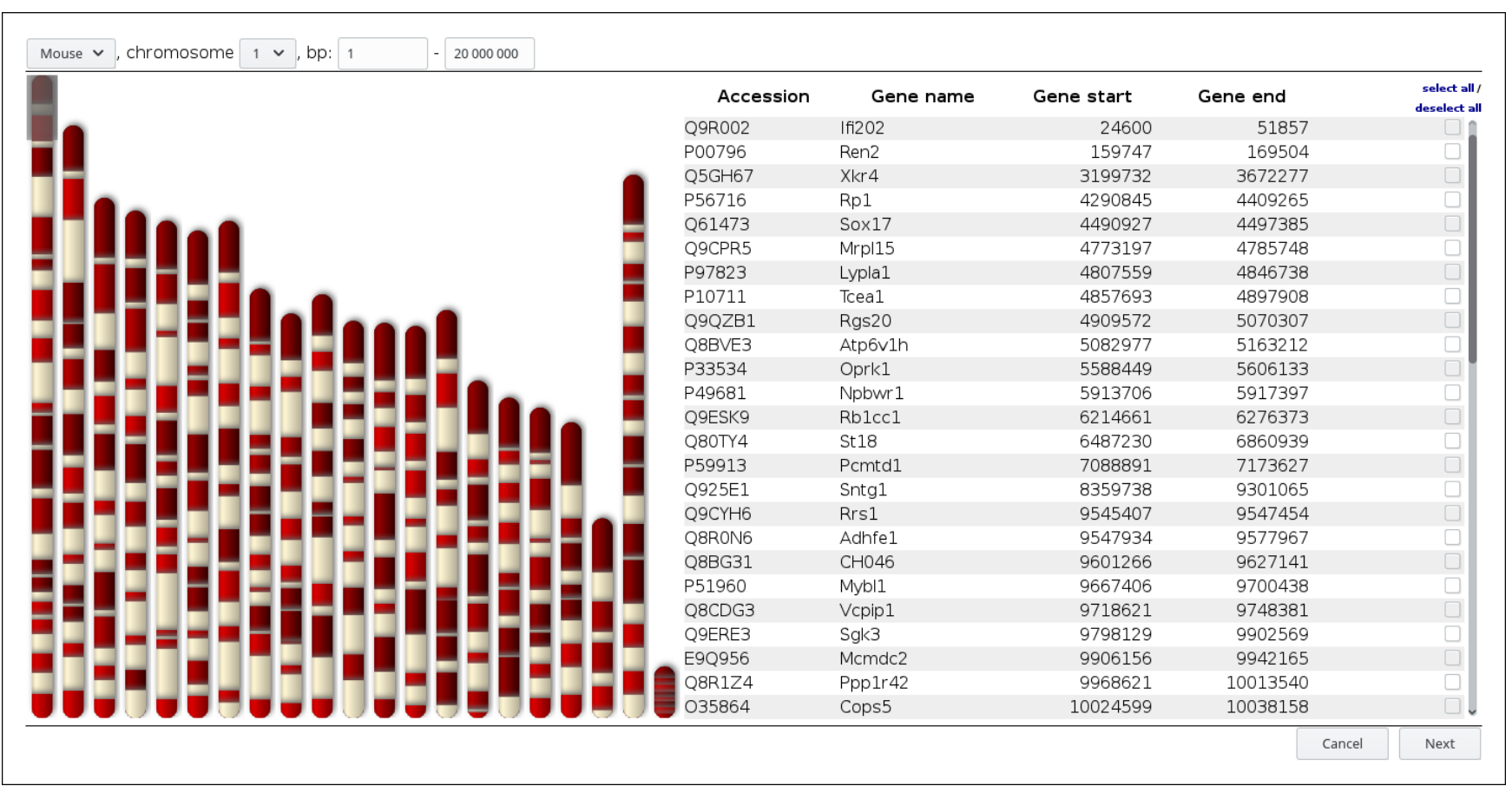

Figure S9 | Chromosome browser for selecting proteins within regions of chromosomes.

\section{Using the pathway editor to create / update / delete pathways}

To offer a simple way for the pathway curators to create, update, or delete pathways, an access-restricted pathway editor was developed. Within this editor it is possible to insert, update, or delete pathway, protein and metabolite information and the according attributes (e.g. pathway name, protein accession number, metabolite EC number etc.). An intuitive drag and drop concept was used to facilitate the pathway editing. 


\section{1) Open the pathway editor}

Open the editor ${ }^{8}$ and type in the credentials. The editor is split into a top menu strip, a tool box with all functions such as buttons on the left hand side and the current view (canvas) on the pathway on the right hand side (consider Figure S10).

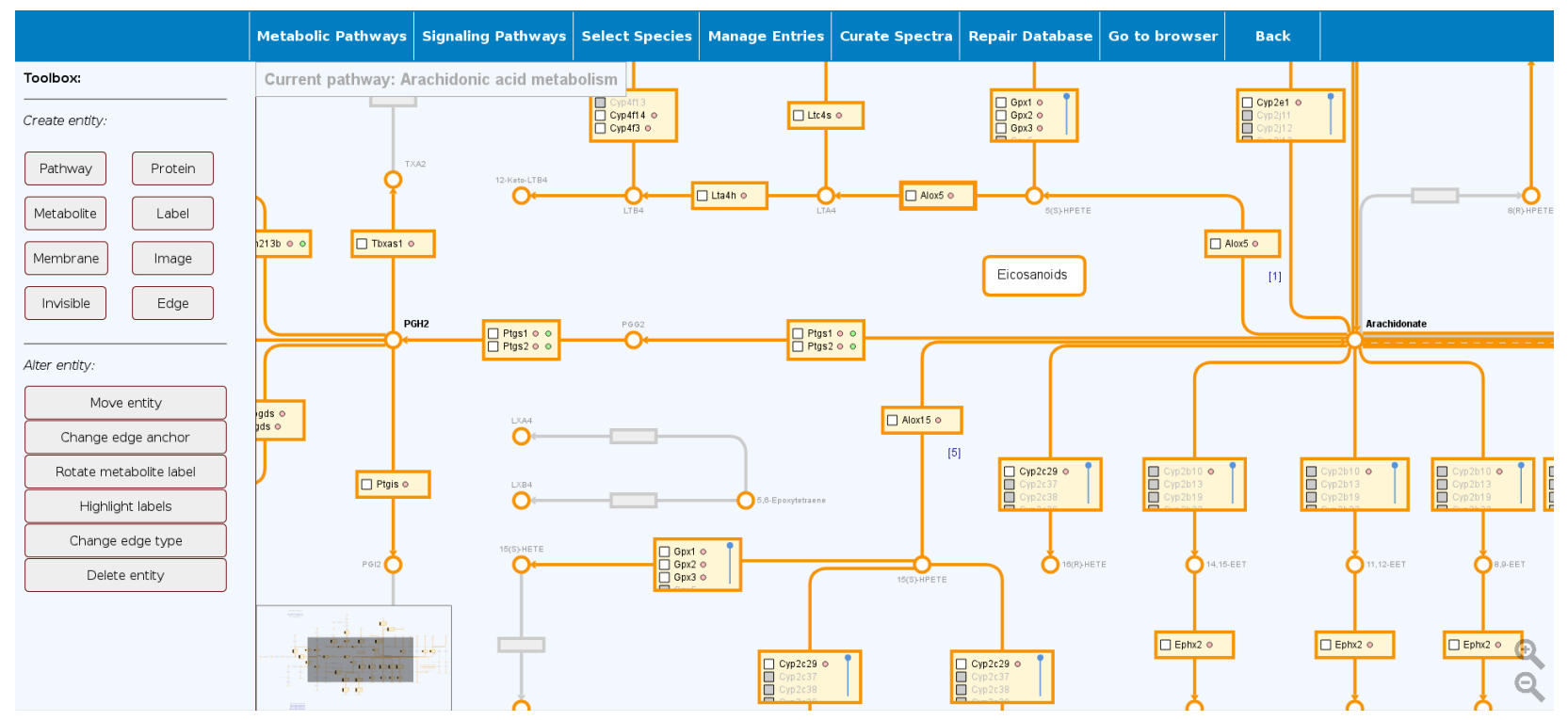

Figure S10 / Basic view on the pathway editor.

The toolbox itself is split into two types of functions $i$ ) for creating visual entities (protein nodes, metabolite nodes, edges, etc.) and ii) for alternating the entities (highlight labels, move entities, etc.). The pathway to be edited can be selected via the buttons "Metabolite Pathways" or "Signaling Pathways" as well as species, respectively.

\section{2) Creating a new pathway}

To create a new pathway which is not yet registered in the database, click on "Manage entries". In the opening window (consider Figure S11), the user can choose to work on pathway group, single pathway, protein or metabolite entries. Pathway entries contain three attributes, i) the pathway name, ii) the membership to a pathway group and iii) a flag if the pathway is a signaling pathway or not. When clicking at the bottom on "New pathway", a dialog is opening requesting those attributes. For instance, one can type in: pathway name = "UPR Signaling", pathway group = "Other pathway", signaling $=$ checked After adding a pathway, its attributes can be furthermore edited in the "Manage entries" window (Figure S11).

\footnotetext{
${ }^{8}$ https://stamps.isas.de/admin
} 


\begin{tabular}{|c|c|c|c|c|c|c|}
\hline \multicolumn{2}{|c|}{ Pathway groups } & Pathways & Proteins & Metabolites & \multirow{2}{*}{\multicolumn{2}{|c|}{ pathway_group_id }} \\
\hline Del & \multicolumn{4}{|c|}{ name 4} & & \\
\hline$x$ & \multicolumn{4}{|c|}{ 3-Chloroacrylic acid degradation } & Other pathways & v \\
\hline$x$ & \multicolumn{4}{|c|}{ Adipocytokine Insignaling pathway } & Other pathways & v \\
\hline$x$ & \multicolumn{4}{|c|}{ Adrenergic signaling in cardiomyocytes } & Other pathways & $\checkmark$ \\
\hline$x$ & \multicolumn{4}{|c|}{ Alanine and aspartate metabolism } & Other pathways & $\checkmark$ \\
\hline$x$ & \multicolumn{4}{|c|}{ Alanine, aspartate and \nglutamate metabolism } & Amino acid metabolism & v \\
\hline$x$ & \multicolumn{4}{|c|}{ Aldosterone synthesis and secretion } & Other pathways & $\checkmark$ \\
\hline$x$ & \multicolumn{4}{|c|}{ Aldosterone-regulatedinsodium reabsorption } & Other pathways & $\checkmark$ \\
\hline$x$ & \multicolumn{4}{|c|}{ alpha-Linolenic acid metabolism } & Lipid metabolism & v \\
\hline$x$ & \multicolumn{4}{|c|}{ Alzheimer disease } & Other pathways & $\checkmark$ \\
\hline$x$ & \multicolumn{4}{|c|}{ Amino sugarln and nucleotideln sugar metabolism } & Carbohydrate metabolism & $\checkmark$ \\
\hline$x$ & \multicolumn{4}{|c|}{ Aminosugars metabolism } & Other pathways & $\checkmark$ \\
\hline$x$ & \multicolumn{4}{|c|}{ AMPK signaling pathway } & Other pathways & $\checkmark$ \\
\hline$x$ & \multicolumn{4}{|c|}{ Apelin signaling pathway } & Other pathways & $\checkmark$ \\
\hline$x$ & \multicolumn{4}{|c|}{ Apoptosis } & Other pathways & $\checkmark$ \\
\hline$x$ & \multicolumn{4}{|c|}{ Arachidonic acid Inmetabolism } & Lipid metabolism & $\checkmark$ \\
\hline$x$ & \multicolumn{4}{|c|}{ Arginine and proline metabolism } & Amino acid metabolism & $\checkmark$ \\
\hline$x$ & \multicolumn{4}{|c|}{ Arginineln biosynthesis } & Amino acid metabolism & $\checkmark$ \\
\hline$x$ & \multicolumn{4}{|c|}{ Ascorbate and $\mathrm{n}$ aldarate metabolism } & Carbohydrate metabolism & $\checkmark$ \\
\hline ra & \multicolumn{4}{|c|}{ A.tankhono. animal } & 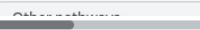 & \\
\hline 12 & $7>$ & New pathw. & & & & \\
\hline
\end{tabular}

Figure S11 / Window for inserting / editing / deleting entries (as pathways, proteins, metabolites) in the database.

\section{3) Inserting entities into the pathway}

Be aware that a recently created pathway is (obviously) empty. Therefore, nothing is visible when selecting the pathway via "Metabolite Pathways" or "Signaling Pathways". Furthermore, in the regular STAMPS pathway browser (Figure S5), empty pathways are not selectable. When clicking on "Protein" in the "Create entity" section of the toolbox, the protein node creation mode is active. Moving over the canvas, an empty protein node is being displayed. When clicking on an arbitrary position on the workspace, a protein node is being spotted on this position and the user can continue to create another protein node. The mode can be deactivated by either clicking once again on "Protein" in the "Create entity" section or selecting another function. Creating pathway nodes, metabolite nodes, labels, or membranes works the same way (Figure S12).

In the next step, the nodes can be filled with entries from the database. Active modes have to be deactivated before. Now, the user can simply click on the nodes and an according pathway, protein, or metabolite selection window appears. Note, that pathway and metabolite nodes are single nodes whereas multiple proteins can be assigned to one protein node. After assigning entries to the node entities, one can rotate the metabolite 
labels by activating the "Rotate metabolite label" mode and clicking on the according metabolite.

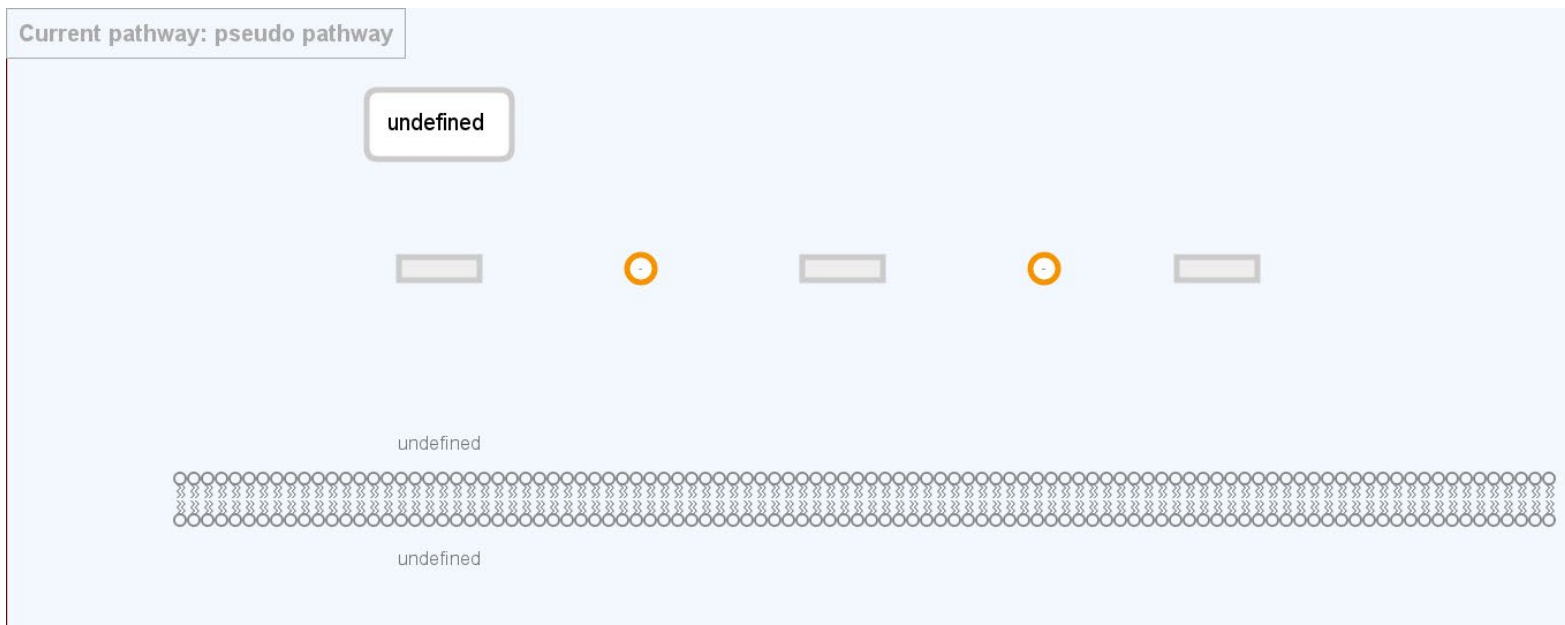

Figure S12 / View on the pathway editor canvas after inserting certain functional nodes.

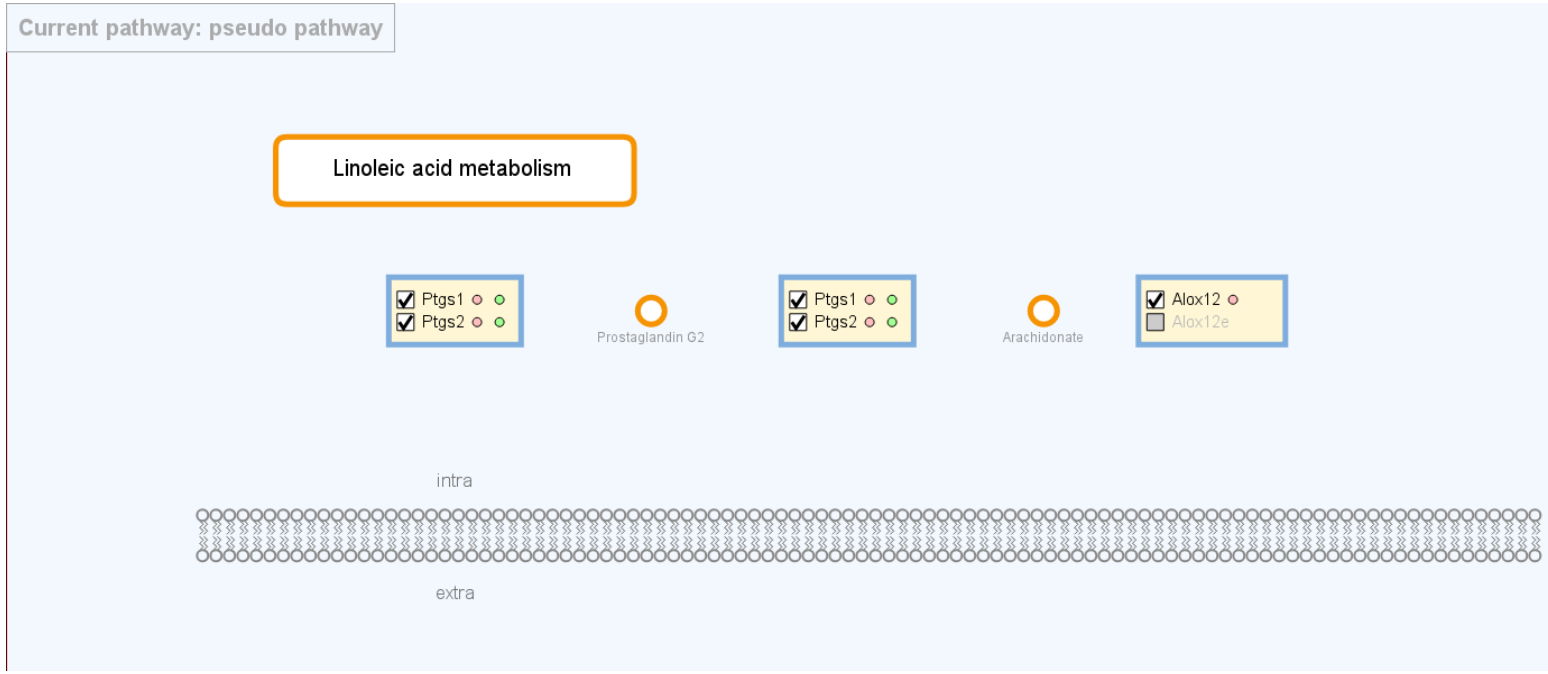

Figure S13 / View on the pathway editor canvas after inserting entries into the according entities.

\section{4) Drawing edges and alter entities}

To draw edges, "Edges" mode in the "Create entity" section must be activated first. Subsequently, anchor points appear for all visual nodes in the canvas. By clicking on one anchor point, holding the mouse button, moving the mouse and releasing over another anchor point, an edge will be drawn and routed automatically. It is allowed to draw edges from each anchor point to each other anchor point, except connecting two anchors of the same node. 
Linoleic acid metabolism

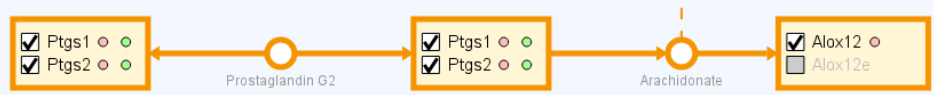

intra

Figure S14 / View on the pathway editor canvas after inserting and decorating edges.

By default, the edges are solid and both their endings are not decorated. When clicking on "Change edge type" and clicking on a certain edge the appearance of the edge is changing with each click. In this way one can create solid and dashed edges as well as add arrows and other symbols on the end of each edge. Figure S14 shows an example. Additional functions are moving node entities and highlighting either metabolite or standalone labels. The last function offers deleting either edges or nodes. When deleting a node, all connected edges will be deleted as well. 


\section{Statistics}

We added several statistical overviews on different levels comprising spectral, protein, and pathway level. Overviews can be accessed over the main page of STAMPS and are dynamically adjustable. The data is taken directly from the protein and spectral database and the figures are always generated on the fly. Figure S15 illustrates all statistical overviews. Shown are e.g. normalized distributions of protein abundances over pathways or over tissues, absolute number of proteins, the normalized spectrum abundance factor, protein isoelectric point versus the protein masses as well as the mass residual distribution between the theoretical spectra and the actually measured precursors. 
a) Normalized distribution of protein abundances per pathway

- Metabolic $\bigcirc$ Signaling sphingolipid metabolism $\vee$

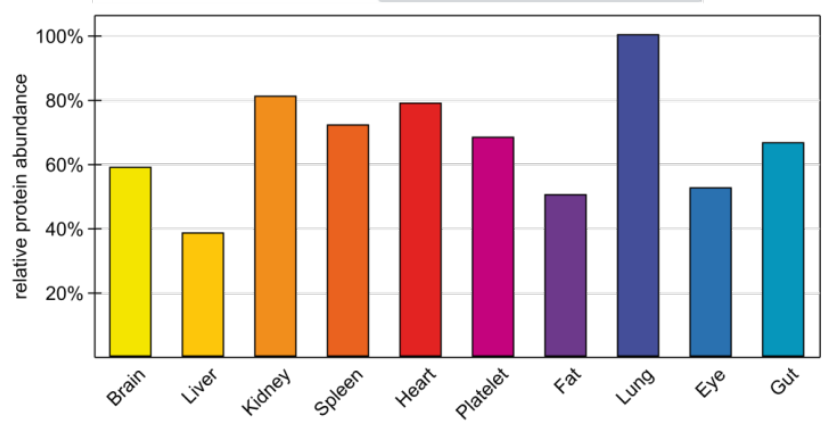

c) Normalized distribution of protein abundances per tissue
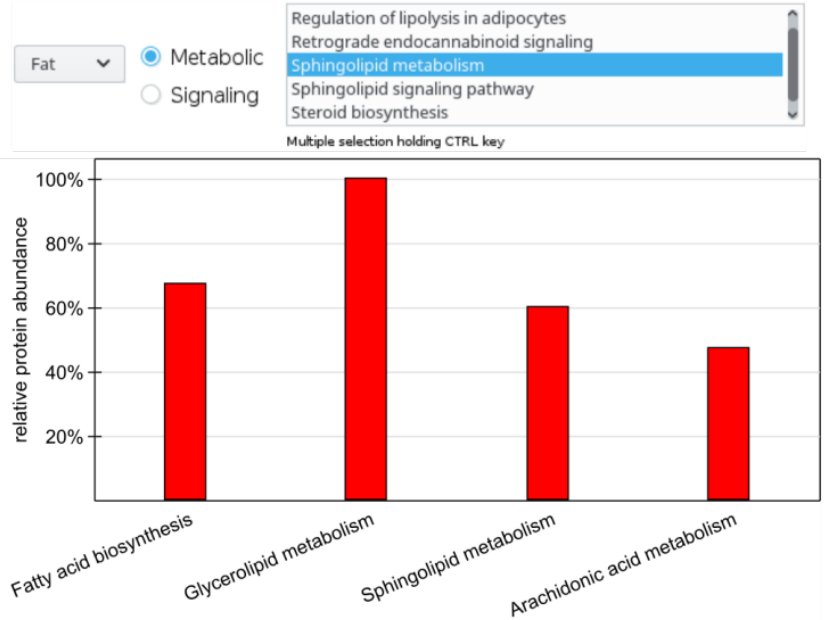

e) Protein isoelectric point vs. mass

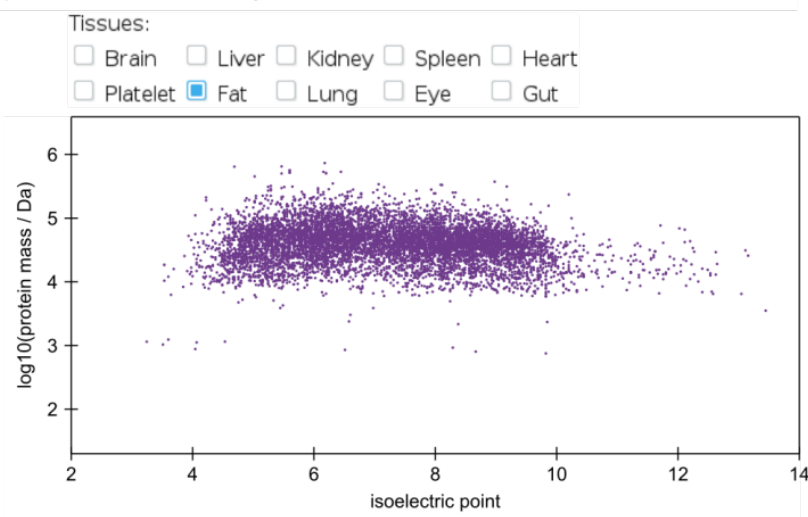

b) Absolute number of proteins

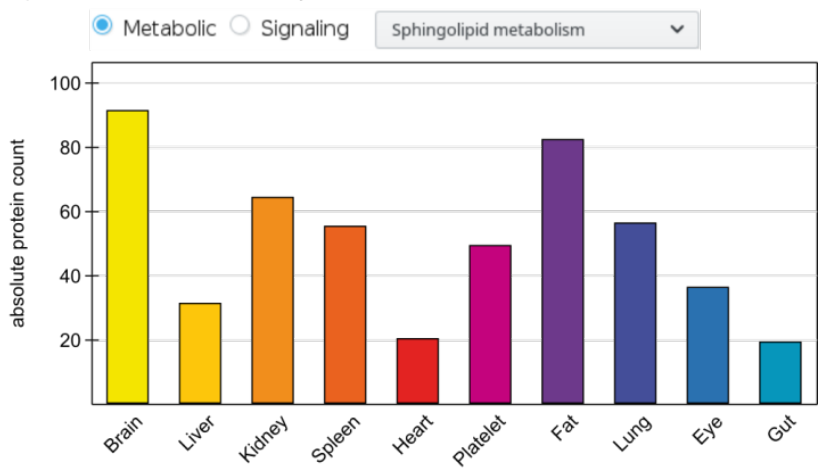

d) Normalized spectrum abundance factor

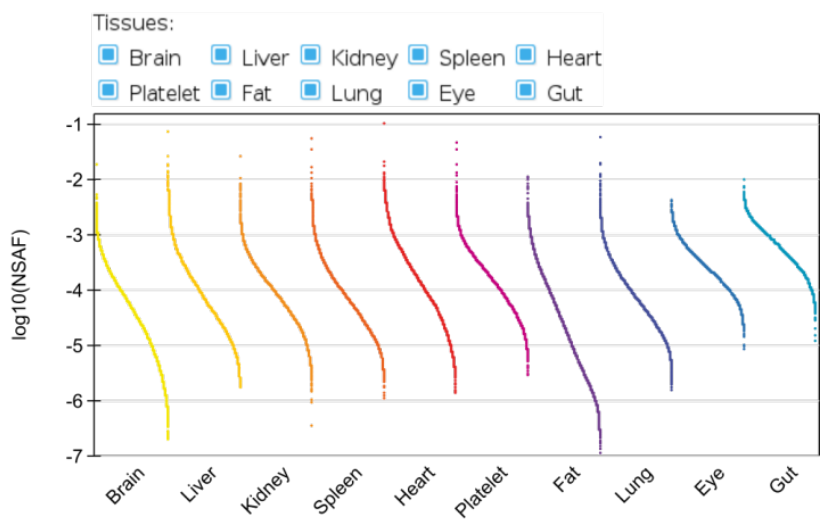

f) Mass accuracy residuals

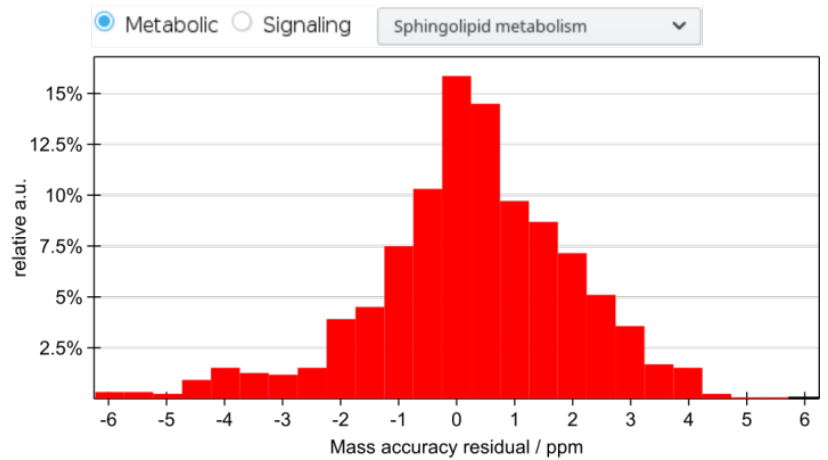

Figure S15 / Statistical overviews. All figures are interactive allowing the user to change parameters.

(1) Kanehisa, M.; Goto, S. Nucleic Acids Research 2000, 28, 27-30.

(2) The UniProt Consortium. Nucleic Acids Research 2017, 45, D158-D169.

(3) Sherry, S. T.; Ward, M.-H.; Kholodov, M.; Baker, J.; Phan, L.; Smigielski, E. M.; Sirotkin, K. Nucleic Acids Research 2001, 29, 308-311. 